\title{
The mechanism of anti-CD20-mediated B cell depletion revealed by intravital imaging
}

\author{
Fabricio Montalvao, ${ }^{1,2}$ Zacarias Garcia, ${ }^{1,2}$ Susanna Celli,1,2 Béatrice Breart,,1,2 \\ Jacques Deguine, ${ }^{1,2}$ Nico Van Rooijen, ${ }^{3}$ and Philippe Bousso ${ }^{1,2}$
}

1 Institut Pasteur, Dynamics of Immune Responses Unit, Paris, France. 2INSERM U668, Paris, France.

${ }^{3}$ Department of Molecular Cell Biology, VU Medical Center, Amsterdam, The Netherlands.

\begin{abstract}
Anti-CD20 Ab therapy has proven successful for treating B cell malignancies and a number of autoimmune diseases. However, how anti-CD20 Abs operate in vivo to mediate B cell depletion is not fully understood. In particular, the anatomical location, the type of effector cells, and the mechanism underlying anti-CD20 therapy remain uncertain. Here, we found that the liver is a major site for $\mathrm{B}$ cell depletion and that recirculation accounts for the decrease in B cell numbers observed in secondary lymphoid organs. Using intravital imaging, we established that, upon anti-CD20 treatment, Kupffer cells (KCs) mediate the abrupt arrest and subsequent engulfment of B cells circulating in the liver sinusoids. KCs were also effective in depleting malignant B cells in a model of spontaneous lymphoma. Our results identify Ab-dependent cellular phagocytosis by KCs as a primary mechanism of anti-CD20 therapy and provide an experimental framework for optimizing the efficacy of therapeutic Abs.
\end{abstract}

\section{Introduction}

Anti-CD20 therapy mediates the depletion of B cells and represents a breakthrough in the treatment of $\mathrm{B}$ cell malignancies and autoimmune disorders (1-3). Multiple underlying mechanisms have been proposed, including complement-dependent cytotoxicity, Ab-dependent cell-mediated cytotoxicity, and Ab-dependent cellular phagocytosis. Preclinical studies have highlighted the importance of Fc receptor-dependent (FcR-dependent) processes for $\mathrm{B}$ cell depletion $(4,5)$, a finding consistent with the observed influence of FCR polymorphism on the efficiency of anti-CD20 therapy in humans (6). Many immune cells, including NK cells, macrophages, and monocytes, kill anti-CD20-coated B cells in vitro and/or are required for depletion in murine models $(4,5,7)$. Paradoxically, despite more than 15 years of clinical experience, the anatomical location, the precise immune cell type, and the mechanism underlying anti-CD20 therapy remain incompletely understood (8). Addressing these questions remains critical to facilitate the design of improved therapeutic Abs. Here, we used a combination of surgical procedures, intravital 2-photon imaging, and a spontaneous tumor model to uncover the mechanism by which anti-CD20 injection results in the clearance of normal and malignant B cells.

\section{Results and Discussion}

To model anti-CD20 therapy, we treated mice with a depleting mouse anti-CD20 Ab (9). After 16 hours, the percentage of $B$ cells was reduced in all organs analyzed, a phenomenon that was strictly dependent on activating Fc receptors (Supplemental Figure 1; supplemental material available online with this article; doi:10.1172/JCI70972DS1). It is noteworthy that after 2 hours B cell depletion was already detectable in the liver but not in secondary lymphoid organs (Figure 1A). To evaluate the contribution of the liver to systemic depletion, mice were subjected to partial hepatectomy, during which $50 \%$ of the liver

Conflict of interest: The authors have declared that no conflict of interest exists. Citation for this article: JClin Invest. 2013;123(12):5098-5103. doi:10.1172/JCI70972. mass was removed. In this setting, the efficiency of systemic anti-CD20-mediated depletion (as measured in the blood) was reduced by approximately half (Figure 1B). In contrast, B cell depletion was unaltered in splenectomized mice (Figure 1B). To test whether blood circulation accounted for the late depletion in secondary lymphoid organs, we exploited the fact that pertussis toxin-treated (PTX-treated) B cells accumulate in the circulation as they fail to enter into lymph nodes and the white pulp of the spleen (ref. 10 and Supplemental Figure 2). In recipients receiving a mixture of untreated and PTX-treated splenocytes, B cells from both subsets were largely depleted in livers by anti-CD20 treatment, but only PTX-treated B cells were efficiently removed from the spleens (Figure 1C). Thus, favoring $B$ cell recirculation increased the efficiency of depletion. Collectively, these results suggest that the liver plays a central role during anti-CD20 therapy.

To characterize the mechanism operating in the liver during antiCD20 treatment, we relied on intravital imaging using mice with RFP-expressing B cells. In untreated animals, we detected B cells circulating in the liver sinusoids, appearing and disappearing rapidly from the imaging volume (Figure 1, D and E; Supplemental Figure 3; and Supplemental Videos 1 and 2). Strikingly, within minutes of anti-CD20 therapy, circulating B cells arrested and remained immotile (Figure 1, D and E, and Supplemental Video 2). After 10 to 30 minutes, the rounded morphology of B cells was lost and RFP fluorescence appeared more diffuse, possibly reflecting $B$ cell death and/or degradation. The liver contains Kupffer cells (KCs), a population of highly phagocytic tissular macrophages, lining the sinusoids and expressing FcRs (11). When mice were injected with clodronate liposomes, a treatment that efficiently removed KCs (Figure 2A), anti-CD20 treatment was no longer effective (Figure $2 \mathrm{~B}$ ). To directly assess the role of $\mathrm{KCs}$, we used a mouse strain expressing a fluorescent reporter for Csfr1 (referred to herein as MAFIA mice; ref. 12), a key regulator of KC survival (13). In the liver, $\mathrm{GFP}^{+}$cells included CD $11 \mathrm{~b}^{\text {hi }} \mathrm{F} 4 / 80^{\text {lo }}$ cells as well as cells with the typical phenotype of KCs, expressing high levels of F4/80 and the bona fide macrophage markers MerTK and CD64 (FcR $\gamma \mathrm{I})$ 
A

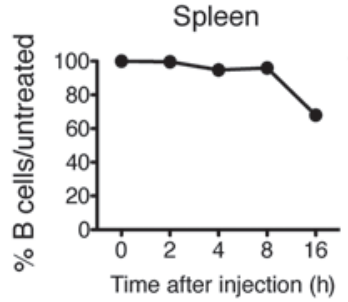

Lymph node

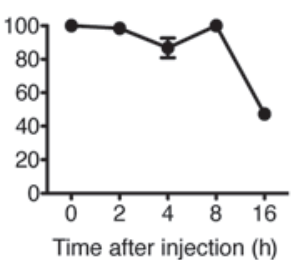

Liver

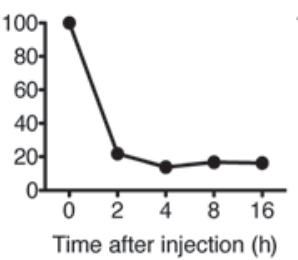

Blood

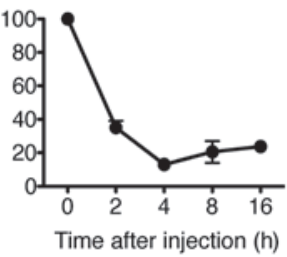

B

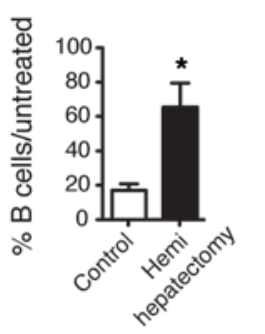

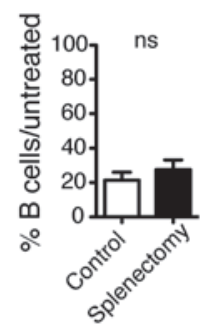

C
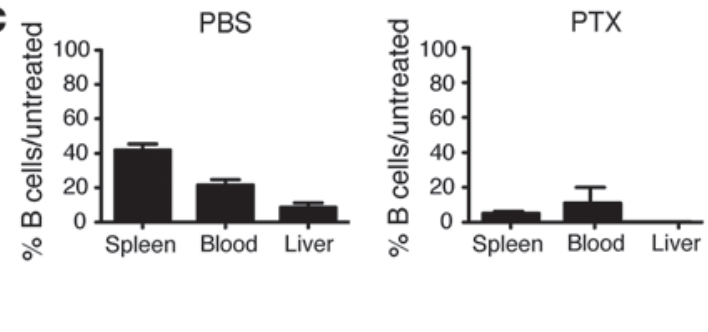

D

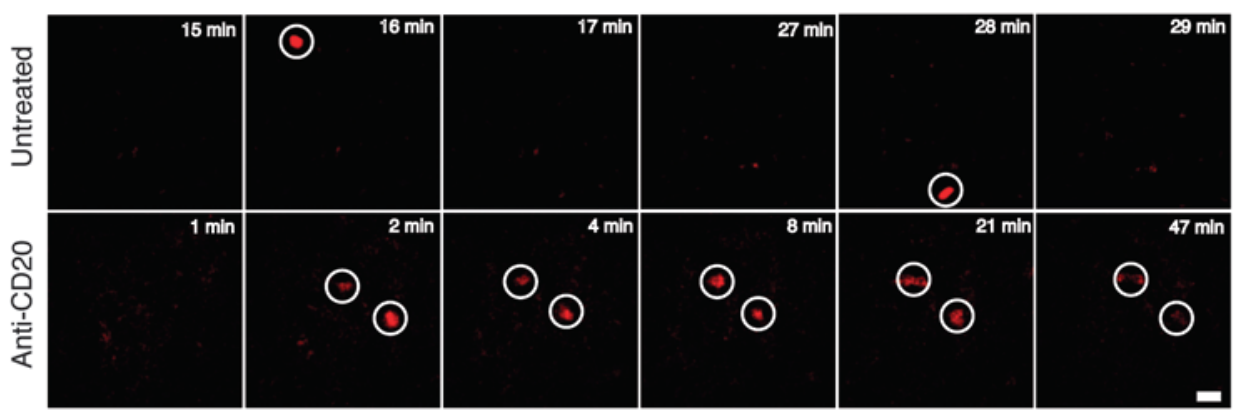

E
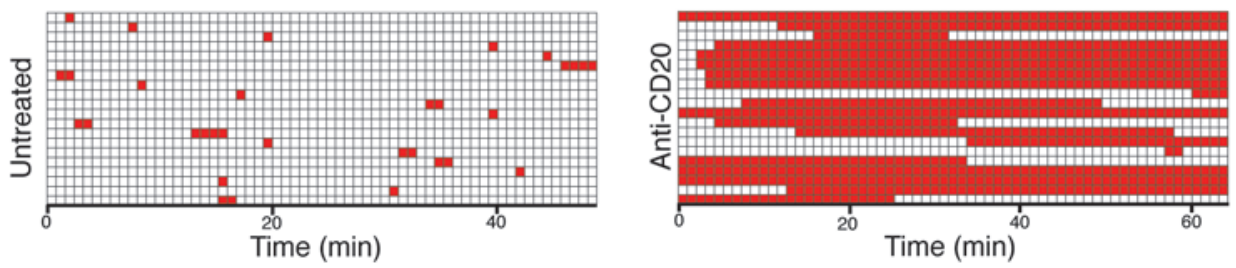

Figure 1

The liver is a major site for anti-CD20-mediated B cell depletion. (A) C57BL/6 mice were treated with a single injection of anti-CD20. The percentage of remaining B cells (compared with that in untreated mice) was measured over time in the indicated organs. (B) Mice were subjected to partial hepatectomy, splenectomy, or left untreated. The efficacy of B cell depletion was calculated as the fraction of B cells remaining in the blood 16 hours after anti-CD20 injection relative to the same values in mice subjected to surgery but not injected with anti-CD20. ${ }^{*} P<0.05$. (C) Recipient Rag2 $2^{-/}$mice were transferred with a 1:1 mixture of untreated and PTX-treated splenocytes and injected with antiCD20. For each subset, the percentage of B cells at 6 hours after anti-CD20 injection (compared with that in recipients that did not receive anti-CD20) is shown for the indicated organs. (D and E) B cells circulating in the liver arrest immediately following anti-CD20 administration. B cells are indicated by white circles. Mice with RFP-expressing B cells were subjected to intravital imaging of the liver. (D) Representative time-lapse images and (E) quantification before or immediately after injection of anti-CD20 Ab. Each horizontal line represents an individual $B$ cell, and red squares represent the time period during which the B cell is visible in the imaging field. Scale bar: $10 \mu \mathrm{m}$.

(Figure 2C). As expected, the latter population was the primary population in the liver targeted by clodronate liposomes (Figure 2D and Supplemental Figure 4). Using in vivo cell labeling with a fluorescent anti-F4/80 Ab and subsequent imaging, we confirmed that $\mathrm{GFP}^{+}$cells expressing the $\mathrm{F} 4 / 80$ marker displayed the typical elongated morphology of KCs and were largely sessile (Figure 2D). $\mathrm{GFP}^{+}$cells that were not labeled by the F4/80 marker were motile and displayed a rounded morphology, corresponding most likely to monocytes and granulocytes (Figure 2E and Supplemental Video 3). Thus, KCs could readily be visualized using the MAFIA mouse model on the basis of GFP expression and cell morphology. We therefore imaged MAFIA mice to monitor B cell interactions with $\mathrm{GFP}^{+} \mathrm{KCs}$. In untreated MAFIA mice, B cells circulating in the liver sinusoids occasionally contacted $\mathrm{KCs}$, but these interactions were transient ( $<5$ minutes) (Figure 3 , A and D, and Supplemental Video 4). Following anti-CD20 injection, virtually all B cells specifically arrested on KCs and were engulfed by the contacting KCs a few minutes later (Figure 3, A and B; Supplemental Video 5; and Supplemental Figure 5A). B cell debris were occasionally seen appearing in KCs, which is suggestive of B cell degradation 
A

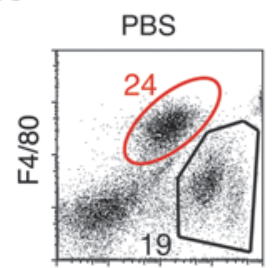

CD11b

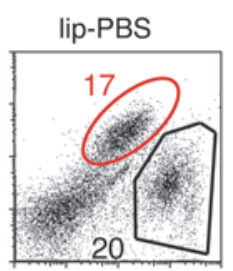

CD11b
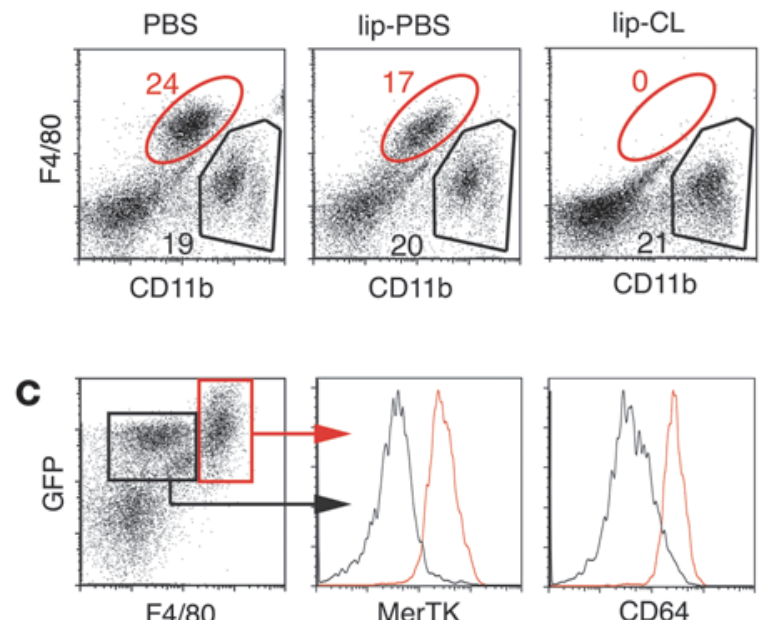

$\mathrm{F} 4 / 80$

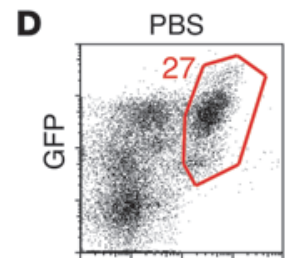

$\mathrm{F} 4 / 80$

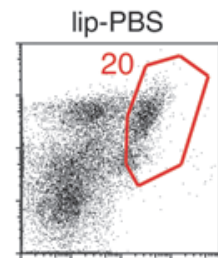

F4/80

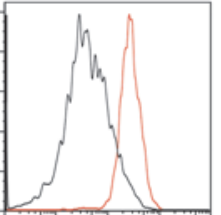

CD64
B

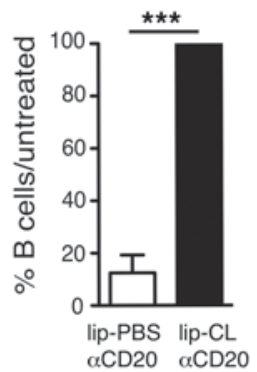

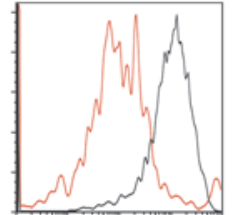

CD11b

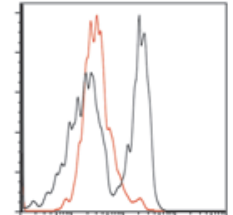

Ly6G

E
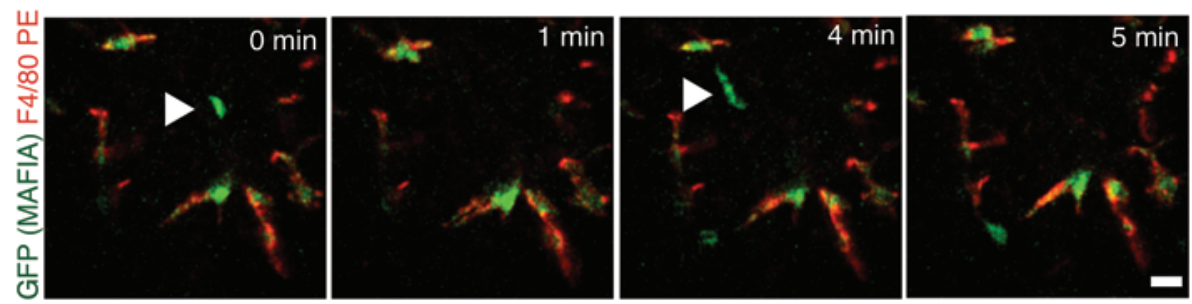

\section{Figure 2}

Tracking KCs using the MAFIA mouse model. (A) Mice were injected intravenously with clodronate (lip-CL) or control liposomes (lip-PBS). After 24 hours, depletion of KCs $\left(\mathrm{F} 4 / 80^{+} \mathrm{CD} 11 \mathrm{~b}^{\mathrm{lo}}\right)$ by lip-CL was confirmed by flow cytometry. Numbers in $\mathbf{A}$ and $\mathbf{D}$ correspond to the percentage of cells falling in the indicated gate. (B) B cell depletion following anti-CD20 injection was quantified as in Figure 1 in the blood in mice treated with clodronate or control liposomes. ${ }^{* *} P<0.001$. (C) Flow cytometric analysis of liver cells in MAFIA mice. Data are gated on $\mathrm{CD}_{45} 5^{+}$cells. Red and black histograms were obtained using the corresponding gates shown in the leftmost panel. (D) MAFIA mice were injected intravenously with clodronate or control liposomes. After 24 hours, liver cells were analyzed by flow cytometry. (E) Sessile GFP+ cells in the livers of MAFIA mice correspond to KCs. MAFIA mice were injected intravenously with PE-conjugated anti$\mathrm{F} 4 / 80 \mathrm{Ab}$ and subjected to intravital imaging of the liver 10 minutes later. Time-lapse images showing that sessile spindle-shaped GFP+ cells with typical $\mathrm{KC}$ morphology were all stained with F4/80 Ab. In contrast, motile GFP+ cells (indicated by white arrowheads) were all F4/80-. Scale bar: $10 \mu \mathrm{m}$.

(Supplemental Figure 5B). B cells were alive when they arrested on KCs and were not simply cleared by KCs after being killed by a distinct effector cell (Supplemental Figure 6). Engulfment of $\mathrm{B}$ cells by liver $\mathrm{F} 4 / 80^{+}$cells was confirmed by immunofluorescence on fixed tissue sections (Supplemental Figure 7). Importantly, B cell arrest upon anti-CD20 treatment was impaired in $\mathrm{FcRY}^{-/}$ recipients (Figure $3 \mathrm{C}$ ), indicating that engagement of activating FcRs on KCs was important for B cell capture.

To test whether KCs also deplete malignant B cells, we relied on the E $\mu$-myc transgenic mice (a model for human Burkitt lymphoma) (14) that spontaneously develop B cell lymphoma.
First, we isolated a fluorescent B cell lymphoma cell line from a E $\mu$-myc transgenic mouse and transferred these cells into MAFIA mice. After 3 weeks, we showed that anti-CD20 injection resulted in the rapid engulfment of lymphoma cells by KCs (Figure 3D and Supplemental Video 6). To test whether a similar mechanism operates in the context of a spontaneously developing lymphoma, we generated Csfr $11^{g f p /+} C d 19^{\text {cre } /+}$ Rosa $26^{R F P /+}$ $E \mu-m y c^{+/-}$mice that developed B cell lymphomas and contained fluorescent KCs and B cells. Intravital imaging revealed that $\mathrm{B}$ cells circulating in liver sinusoids were rapidly engulfed by KCs upon anti-CD20 injection (Figure 3, E and F). Thus, KCs 
A

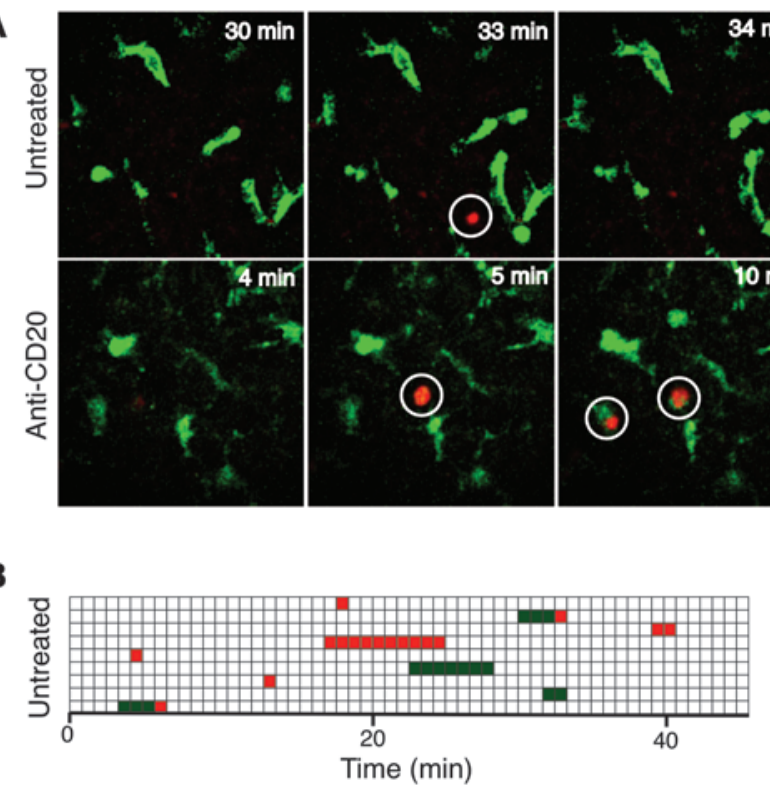

C

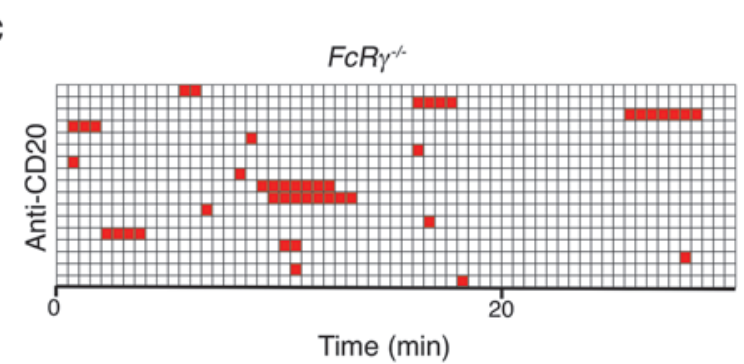

Time (min)

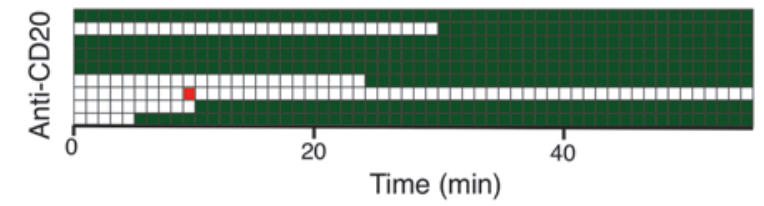

D

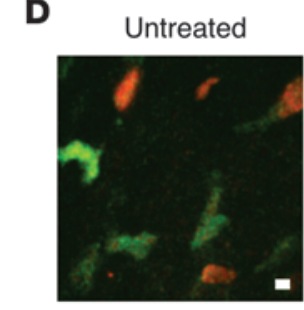

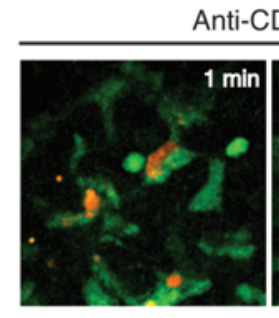

Tumor B cell line
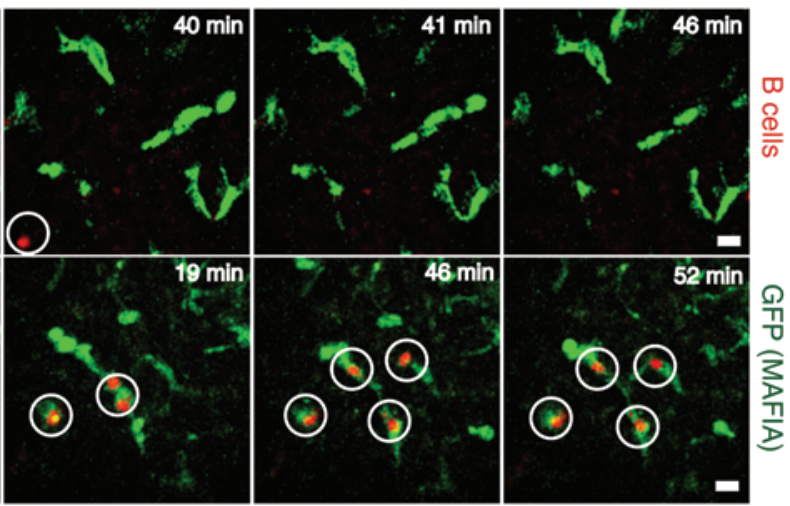

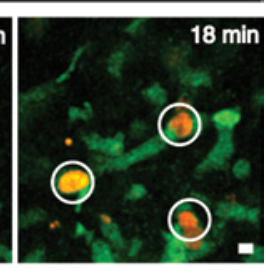

GFP (MAFIA)
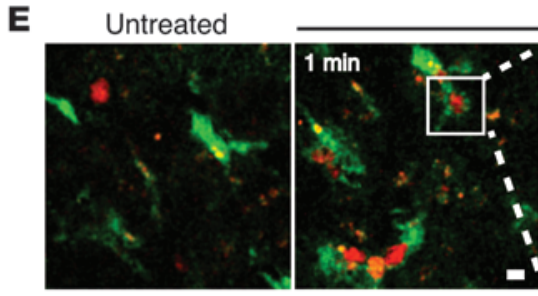

Endogenous E $\mu$-Myc B cells
Anti-CD20

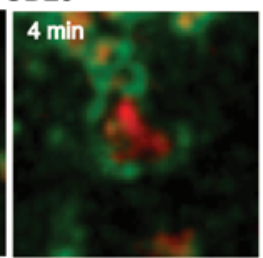

GFP (MAFIA)
F

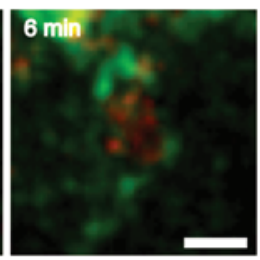

Anti-CD20

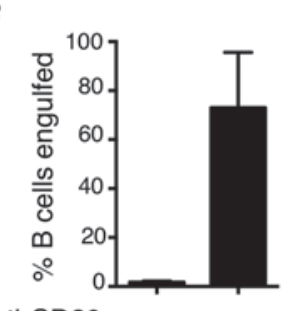

$+$

\section{Figure 3}

KCs mediate the arrest and engulfment of normal and malignant B cells during anti-CD20 therapy. (A-D) Rag2-/- MAFIA mice were adoptively transferred with dye-labeled B cells and subjected to intravital imaging of the liver. (A) Representative time-lapse images recorded before or immediately after anti-CD20 injection. B cells are indicated by white circles. (B) B cell behavior and contact with KCs before or after anti-CD20 injection. Each line represents an individual B cell, red squares represent the time period during which B cells are present in the imaging field without contacting $\mathrm{KCs}$, and green squares indicate the time period during which $\mathrm{B}$ cells are associated with a KC. (C) Impaired B cell arrest in $F c R \gamma^{-1-}$ mice treated with anti-CD20. Rag $2^{-1-} F c R \gamma^{-/-}$mice were transferred with dye-labeled B cells and injected with anti-CD20. (D) Rag2 ${ }^{-1-}$ MAFIA mice were injected with a YFP-expressing B cell tumor line. Representative time-lapse images show that tumor cells were rapidly engulfed by KCs upon anti-CD20 injection. Engulfed B cell tumors are indicated by white circles. (E and $\mathbf{F})$ KCs are effectors of anti-CD20 therapy in a model of spontaneously developing B cell lymphoma. Csfr 1 gfp $/+C d 19^{\text {cre } /+R o s a 26 R F P /+} E \mu-m y C^{+/-}$mice were subjected to intravital imaging of the liver following anti-CD20 injection. (E) Representative time-lapse images showing the engulfment and subsequent digestion of RFP-expressing lymphoma cells by KCs. (F) Quantification of B cell engulfment by KCs following anti-CD20 therapy. Scale bar: $10 \mu \mathrm{m}$.

mediate the elimination of malignant B cells during anti-CD20 therapy in a physiological model of B cell lymphoma.

In the present study, we address a long-standing question and establish the mode of action of anti-CD20 in a mouse model.
Our results demonstrate that the liver is a major site for depletion and extend the results of a previous study, highlighting the importance of B cell circulation for systemic depletion (15). Most importantly, intravital imaging revealed that $\mathrm{KCs}$ 
were responsible for B cell depletion through Ab-dependent cellular phagocytosis. In humans, the liver contains $15 \%$ of total blood volume and filters one-third of the cardiac output. Positioned in proximity to hepatic sinusoids, KCs are ideally located to capture circulating Ab-coated B cells (16). Following $\mathrm{mAb}$ injection, previous studies have observed target cells within KCs on fixed tissue sections $(15,17)$. Whether this reflected the depletion mechanism or whether KCs were simply scavenging dead cells previously killed by other effectors could not be directly addressed. Here, with the help of dynamic imaging, we established that $\mathrm{B}$ cell depletion proceeds in two steps. First, within minutes of anti-CD20 injection, circulating B cells abruptly arrested on KCs. Subsequently, $B$ cells were engulfed and degraded by the contacting KCs. Overall, this process was remarkably efficient, as virtually all liver B cells were trapped within minutes of anti-CD20 injection. These findings have important implications for the use and development of anti-CD20 therapy. First, we would predict that depletion of malignant lymphoid cells would be more efficient for recirculating tumor cells than for cells trapped in secondary lymphoid organs. Second, our imaging approach should help define $\mathrm{Ab}$ modifications that increase the rate of $B$ cell arrest and/or engulfment by KCs. In preclinical models, the efficacy of different type I anti-CD20 mAbs, like rituximab and ofatumumab, largely depends on FcRs $(4,18,19)$. Thus, the mechanism identified in the present report likely pertains to other anti-CD20 Abs that largely rely on FcRs to induce depletion $(4,5,15,19)$. Interestingly, type II antiCD20 Abs have been shown to exert direct cytotoxicity against B cells $(20,21)$. Future imaging studies combined with humanized mouse models should therefore help generalize our findings and clarify the contribution of direct versus FcR-dependent effects mediated by type II anti-CD20 Ab in vivo.

In summary, we have established here that Ab-dependent cellular phagocytosis by KCs is a primary mechanism of anti-CD20 therapy and have illustrated how intravital imaging can be used to uncover the mode of action of therapeutics drugs.

\section{Methods}

Further information can be found in the Supplemental Methods.

Mice. E $\mu$-myc transgenic (14) and Csfr1sp/++ (also referred to as MAFIA; ref. 12) mice were purchased from The Jackson Laboratory. C57BL/6, Rag2 $2^{-/}$, Rosa26 $6^{\text {RFP/+ }}, C d 19^{\text {cre/cre }}, F c R \gamma^{-/}$, and Ubi-GFP mice were bred in our animal facility $(22,23)$.

Flow cytometry. Livers were perfused with $8 \mathrm{ml}$ PBS. Organs were incubated for 30 minutes at $37^{\circ} \mathrm{C}$ in RPMI containing $0.5 \mathrm{mg} / \mathrm{ml}$ collagenase. Cell suspensions were Fc blocked using anti-CD16/32 Ab (eBioscience). Analyses were performed using a FACSCanto II Cytometer (BD Biosciences) and analyzed using FlowJo software version 8.6.6 (Tree Star).

Anti-CD20 treatments. Mice were treated with one single dose of 50 or 100 $\mu \mathrm{g}$ mouse anti-mouse CD20 (clone 5D2, isotype IgG2a, Genentech) to evaluate depletion of endogenous or malignant B cells, respectively. For B cell transfer, splenic B cells were isolated by negative selection (Miltenyi Biotech), loaded with $10 \mu \mathrm{M}$ SNARF or CellTracker Orange (Invitrogen), and injected intravenously $\left(0.5 \times 10^{8}\right.$ to $1 \times 10^{8}$ cells). Pretreatment with PTX (Sigma-Aldrich) was performed by incubating splenocytes isolated from GFP-expressing mice $\left(3 \times 10^{7}\right.$ cells $\left./ \mathrm{ml}\right)$ at $37^{\circ} \mathrm{C}$ for 2 hours in RPMI containing $2 \% \mathrm{FCS}$ and $200 \mathrm{ng} / \mathrm{ml} \mathrm{PTX}$. GFP- splenocytes isolated from WT mice were left untreated. Cells were transferred at a 1:1 ratio into Rag2-/recipients. Clodronate was encapsulated in liposomes as described earlier and injected intravenously (24).

Two-photon intravital imaging. Two-photon imaging was performed as previously described (25) with some modifications. In brief, we modified an upright DM6000B microscope (Leica Microsystems) with a custom-designed objective inverter (for use as an inverted microscope) and a $\times 40 / 1.3 \mathrm{NA}$ oil-dipping objective (Nikon). Excitation was provided by a Chameleon Ultra Ti:Sapphire Laser (Coherent) tuned at 1,000 nm. Mice were anesthetized, and the liver was exposed through a horizontal incision in the mid-abdomen, carefully pulled down, and glued onto a glass bottom petri dish heated to $36^{\circ} \mathrm{C}$. Mice were placed onto a heated stage and provided with $95 \%$ oxygen. Data sets were processed and analyzed using Imaris (version 7.2, Bitplane) and ImageJ (version 1.46) softwares.

Statistics. Data are shown as mean \pm SEM and were analyzed using a 2-tailed unpaired Student's $t$ test.

Study approval. Animal experiments wereperformed with the approval of Institut Pasteur and in accordance with its guidelines for animal care and use.

\section{Acknowledgments}

We thank F. Lemaître for help in the generating cell lines. This work was supported by Institut Pasteur, INSERM, the Fondation pour la Recherche Médicale, and an European Research Council starting grant (LymphocyteContacts).

Received for publication May 10, 2013, and accepted in revised form August 22, 2013.

Address correspondence to: Philippe Bousso, Dynamics of Immune Responses Unit, Institut Pasteur, 25 rue du Dr Roux, 75015 Paris, France. Phone: 33.1.45.68.85.51; Fax: 33.1.45.68.84.35; E-mail: philippe.bousso@pasteur.fr.
1. Maloney DG. Anti-CD20 antibody therapy for B-cell lymphomas. NEngl J Med. 2012;366(21):2008-2016.

2. Chan AC, Carter PJ. Therapeutic antibodies for autoimmunity and inflammation. Nat Rev Immunol. 2010;10(5):301-316.

3. Weiner LM, Surana R, Wang S. Monoclonal antibodies: versatile platforms for cancer immunotherapy. Nat Rev Immunol. 2010;10(5):317-327.

4. Clynes RA, Towers TL, Presta LG, Ravetch JV. Inhibitory $\mathrm{Fc}$ receptors modulate in vivo cytotoxicity against tumor targets. Nat Med. 2000;6(4):443-446.

5. Uchida J, et al. The innate mononuclear phagocyte network depletes B lymphocytes through Fc receptor-dependent mechanisms during antiCD20 antibody immunotherapy. J Exp Med. 2004; 199(12):1659-1669.

6. Cartron G, et al. Therapeutic activity of human- ized anti-CD20 monoclonal antibody and polymorphism in IgG Fc receptor FcgammaRIIIa gene. Blood. 2002;99(3):754-758.

7. Biburger M, et al. Monocyte subsets responsible for immunoglobulin G-dependent effector functions in vivo. Immunity. 2011;35(6):932-944.

8. Boross P, Leusen JH. Mechanisms of action of CD20 antibodies. Am J Cancer Res. 2012;2(6):676-690.

9. Sarikonda G, et al. Transient B-cell depletion with anti-CD20 in combination with proinsulin DNA vaccine or oral insulin: immunologic effects and efficacy in NOD mice. PLOS ONE. 2013; 8(2):e54712.

10. Cyster JG, Goodnow CC. Pertussis toxin inhibits migration of $\mathrm{B}$ and $\mathrm{T}$ lymphocytes into splenic white pulp cords. J Exp Med. 1995;182(2):581-586.

11. Ganesan LP, et al. FcgammaRIIb on liver sinusoi- dal endothelium clears small immune complexes. J Immunol. 2012;189(10):4981-4988.

12. Burnett $\mathrm{SH}$, et al. Conditional macrophage ablation in transgenic mice expressing a Fas-based suicide gene. J Leukoc Biol. 2004;75(4):612-623.

13. MacDonald KP, et al. An antibody against the colony-stimulating factor 1 receptor depletes the resident subset of monocytes and tissue- and tumorassociated macrophages but does not inhibit inflammation. Blood. 2010;116(19):3955-3963.

14. Harris AW, Pinkert CA, Crawford M, Langdon WY, Brinster RL, Adams JM. The E mu-myc transgenic mouse. A model for high-incidence spontaneous lymphoma and leukemia of early B cells. J Exp Med. 1988;167(2):353-371.

15. Gong Q, et al. Importance of cellular microenvironment and circulatory dynamics in B cell immu- 
notherapy. J Immunol. 2005;174(2):817-826.

16. Nemeth E, Baird AW, O'Farrelly C. Microanatomy of the liver immune system. Semin Immunopathol. 2009;31(3):333-343.

17. van der Bij GJ, et al. Experimentally induced liver metastases from colorectal cancer can be prevented by mononuclear phagocyte-mediated monoclonal antibody therapy. J Hepatol. 2010;53(4):677-685.

18. de Haij S, et al. In vivo cytotoxicity of type I CD20 antibodies critically depends on Fc receptor ITAM signaling. Cancer Res. 2010;70(8):3209-3217.

19. Chao MP, et al. Anti-CD47 antibody synergizes with rituximab to promote phagocytosis and eradicate non-Hodgkin lymphoma. Cell. 2010; 142(5):699-713.

20. Mossner E, et al. Increasing the efficacy of CD20 antibody therapy through the engineering of a new type II anti-CD20 antibody with enhanced direct and immune effector cell-mediated B-cell cytotoxicity. Blood. 2010;115(22):4393-4402.

21. Glennie MJ, French RR, Cragg MS, Taylor RP. Mechanisms of killing by anti-CD20 monoclonal antibodies. Mol Immunol. 2007;44(16):3823-3837.

22. Luche H, Weber O, Nageswara Rao T, Blum C, Fehling HJ. Faithful activation of an extra-bright red fluorescent protein in "knock-in" Cre-reporter mice ideally suited for lineage tracing studies. Eur J Immunol. 2007;37:43-53.

23. Rickert RC, Roes J, Rajewsky K. B lymphocyte-specific, Cre-mediated mutagenesis in mice. Nucleic Acids Res. 1997;25(6):1317-1318.

24. Van Rooijen N, Sanders A. Liposome mediated depletion of macrophages: mechanism of action, preparation of liposomes and applications. J Immunol Methods. 1994;174(1-2):83-93.

25. Celli S, Lemaitre F, Bousso P. Real-time manipulation of T cell-dendritic cell interactions in vivo reveals the importance of prolonged contacts for CD4+ T cell activation. Immunity. 2007;27(4):625-634. 